NBER WORKING PAPER SERIES

IS BITCOIN A REAL CURRENCY? AN ECONOMIC APPRAISAL

David Yermack

Working Paper 19747

http://www.nber.org/papers/w19747

\author{
NATIONAL BUREAU OF ECONOMIC RESEARCH \\ 1050 Massachusetts Avenue \\ Cambridge, MA 02138 \\ December 2013
}

For helpful comments I thank audience members at the 2013 annual meeting of the Southern Finance Association and my colleagues Geoffrey Miller, Eric Posner, Roy Smith and Richard Sylla. Part of this research was completed while I was a visiting professor at Erasmus University Rotterdam. The views expressed herein are those of the author and do not necessarily reflect the views of the National Bureau of Economic Research.

NBER working papers are circulated for discussion and comment purposes. They have not been peerreviewed or been subject to the review by the NBER Board of Directors that accompanies official NBER publications.

(C) 2013 by David Yermack. All rights reserved. Short sections of text, not to exceed two paragraphs, may be quoted without explicit permission provided that full credit, including $\mathbb{C}$ notice, is given to the source. 
Is Bitcoin a Real Currency? An economic appraisal

David Yermack

NBER Working Paper No. 19747

December 2013, Revised April 2014

JEL No. E42,G23

\begin{abstract}
$\underline{\text { ABSTRACT }}$
A bona fide currency functions as a medium of exchange, a store of value, and a unit of account, but bitcoin largely fails to satisfy these criteria. Bitcoin has achieved only scant consumer transaction volume, with an average well below one daily transaction for the few merchants who accept it. Its volatility is greatly higher than the volatilities of widely used currencies, imposing large short-term risk upon users. Bitcoin's daily exchange rates exhibit virtually zero correlation with widely used currencies and with gold, making bitcoin useless for risk management and exceedingly difficult for its owners to hedge. Bitcoin prices of consumer goods require many decimal places with leading zeros, which is disconcerting to retail market participants. Bitcoin faces daily hacking and theft risks, lacks access to a banking system with deposit insurance, and it is not used to denominate consumer credit or loan contracts. Bitcoin appears to behave more like a speculative investment than a currency.
\end{abstract}

David Yermack

Stern School of Business

New York University

44 West Fourth Street, Suite 9-160

New York, NY 10012

and NBER

dyermack@stern.nyu.edu 


\section{Is bitcoin a real currency? \\ An economic appraisal}

\section{Introduction}

Bitcoin became a fixture in world financial news in late 2013 and early 2014. The “virtual currency" had been launched five years earlier by computer hobbyists, and in late 2013 the U.S. dollar exchange rate for one bitcoin rose more than fivefold in the space of a few weeks. The market value of one bitcoin, which had begun trading at less than five cents in 2010, briefly exceeded $\$ 1,200.00$. Two days of hearings were held by the U.S. Senate Committee on Homeland Security and Governmental Affairs, and government regulators testified that algorithmic, stateless currencies like bitcoin had the potential to play useful roles in the commercial payment system. ${ }^{1}$ Stories appeared in the media about travelers subsisting for lengthy periods by spending only bitcoin, and various businesses, some of them exotic such as Richard Branson's Virgin Galactic space travel, attracted publicity by accepting bitcoin as payment.

The euphoric news surrounding bitcoin at the end of 2013 gave way to catastrophe in

\footnotetext{
${ }^{1}$ U.S. Senate Committee on Homeland Security and Governmental Affairs, Beyond Silk Road: Potential Risks, Threats, and Promises of Virtual Currencies, Hearings held November 18-19, 2013, available at hsgac.senate.gov/hearings/beyond-silk-road-potential-risks-threats-and-promises-of-virtual-currencies.
} 
February 2014, when the Mt. Gox exchange, once the leader in worldwide bitcoin trading, imploded in a spectacular bankruptcy. Hundreds of millions of dollars worth of bitcoins went missing in connection with the failure of Mt. Gox, yet the value of bitcoins on other exchanges remained surprisingly high at around $\$ 450$ each at the time of this writing. Figure 1 shows the daily closing dollar-bitcoin exchange rate on the Mt. Gox exchange up until February 2014, and thereafter on the Bitstamp exchange, which took over the top spot in trading volume after Mt. Gox folded.

In this paper, I examine whether bitcoin should be considered a currency, an issue that has drawn increasing attention from market regulators concerned about the tax, insurance, and other consequences of how bitcoin is treated legally. I argue that bitcoin does not behave much like a currency according to the criteria widely used by economists. Instead bitcoin resembles a speculative investment similar to the Internet stocks of the late 1990s.

Money is typically defined by economists as having three attributes: it functions as a medium of exchange, a unit of account, and a store of value. Bitcoin somewhat meets the first of these criteria, because a growing number of merchants, especially in online markets, appear willing to accept it as a form of payment. However, the worldwide commercial use of bitcoin remains minuscule, indicating that few people use it widely as a medium of exchange. I further argue in the sections below that bitcoin performs poorly as a unit of account and as a store of value. Bitcoin requires merchants to quote the prices of common retail goods out to four or five decimal places with leading zeros, a practice rarely seen in consumer marketing and likely to confuse both sellers and buyers in the marketplace. Bitcoin exhibits very high time series volatility and trades for different prices on different exchanges without the possibility of 
arbitrage. All of these characteristics tend to undermine bitcoin's usefulness as a unit of account. As a store of value, bitcoin faces great challenges due to rampant hacking attacks, thefts, and other security-related problems. Bitcoin's daily exchange rate with the U.S. dollar exhibits virtually zero correlation with the dollar's exchange rates against other prominent currencies such as the euro, yen, Swiss franc, or British pound, and also against gold. Therefore bitcoin's value is almost completely untethered to that of other currencies, which makes its risk nearly impossible to hedge for businesses and customers and renders it more or less useless as a tool for risk management.

Bitcoin lacks additional characteristics that are usually associated with currencies in modern economies. Bitcoin cannot be deposited in a bank, and instead it must be possessed through a system of "digital wallets" that have proved both costly to maintain and vulnerable to predators. No form of insurance has been developed for owners of bitcoin comparable to the deposit insurance relied on by bank customers in most economies. No lenders use bitcoin as the unit of account for standard consumer finance credit, auto loans, and mortgages, and no credit or debit cards have been denominated in bitcoin. Bitcoin cannot be sold short, and financial derivatives such as forward contracts and swaps that are routine for other currencies do not exist for bitcoin. The absence of these types of market-correcting arbitrage vehicles seems to be the most straightforward explanation for the endurance of bitcoin's value in recent months.

The remainder of this paper is organized as follows. Section II describes the history and background of bitcoin. Section III analyzes whether bitcoin fulfills the classical criteria of a currency. Section IV discusses obstacles faced by bitcoin and concludes the paper. 


\section{History and background of bitcoin}

For much of the $19^{\text {th }}$ and $20^{\text {th }}$ centuries, the world's most successful currencies were convertible into fixed amounts of gold or other precious metals, and for thousands of years prior to that, many currencies were minted directly from gold or silver specie. This direct connection between money and gold, secured by sovereign inventories such as the Fort Knox depository in the U.S., created public confidence in a currency's value. The gold standard collapsed in most economies between the 1920s and 1970s, partly due to the pressures of financing two World Wars, but probably even more because worldwide production of gold did not keep pace with economic growth. Since then, nearly every major economy has issued paper fiat currency, the value of which relies on public belief that a nation's government or central bank will not increase the supply of new banknotes too rapidly. Multinational consortia have issued currency like the euro on similar terms. Fiat currencies have circulated for thousands of years, and sooner or later nearly all of them have been inflated down to worthlessness by governments confronted by strained public finances.

Bitcoin attempts to overcome the weaknesses of both fiat and gold-based money, functioning as an algorithmic currency with a deterministic supply and growth rate tied to the rigor of mathematics. No government or other central authority can manipulate the supply of bitcoins. Instead the currency is governed by cryptographic rules that are enforced by transparent computer code in a decentralized manner. While some enthusiasts have suggested a connection between bitcoin's algorithmic growth rate and the monetary orthodoxy espoused by Milton Friedman, the bitcoin protocol appears to give little or no attention to any optimal rate of monetary growth. Instead, it provides for the rate of seigniorage to slow asymptotically to zero 
by the year 2140, when the last bitcoin is scheduled to be released and the final total will be fixed at 21 million units.

While the limited supply of bitcoins seems to be a critical aspect of the currency's appeal, whether the supply is truly fixed has become a matter of some disagreement. The case against this view is stated by bitcoin entrepreneur in a recent interview with Goldman Sachs (2014):

[I]f you needed to create more, you could. That would require $51 \%$ of the computing power of the network to switch their software to adopt the change. Changes to the software have occurred a couple of times in the past. There are developer forums where such types of changes are typically discussed and a consensus is ultimately reached across the mining community that maintains the network.

If Ersham's interpretation is correct, it implies that the growth of bitcoins could be adapted for reasons of monetary policy, with this macroeconomic policy decisions controlled by an online discussion forum or blog rather than by an expert agency such as the Federal Reserve

Bitcoin originated using a scheme outlined in Nakamoto (2008), a nine page proposal for a "peer-to-peer electronic cash system." The author or authors of this document have not been identified, ${ }^{2}$ but their system was designed in a way that gave them no royalties or residual property rights to benefit from bitcoin's adoption. According to the algorithms proposed by Nakamoto, new bitcoins are created and awarded to computer users who solve pre-specified mathematical problems. These problems become more difficult and less frequent over time. A transparent, decentralized registry tracks the ownership and subsequent transfers of every bitcoin after it is "mined" by its initial owners. All of the quantities and growth rates of bitcoins are known with certainty by the public, so its circulation cannot be affected by monetary policy in

\footnotetext{
${ }^{2}$ A controversial Newsweek story on March 6, 2014, claimed to have located and identified Nakomoto, but the magazine's claim was denied by the subject of the story and has been subject to continuing uncertainty. See http://mag.newsweek.com/2014/03/14/bitcoin-satoshi-nakamoto.html.
} 
the way that the Federal Reserve controls the growth rate of the public supply of U.S. dollars.

Wallace (2011) reviews the early history of bitcoin and states that Nakamoto mined and introduced the first 50 units into circulation in 2009 , essentially to demonstrate the method to a group of online observers. Bitcoin's circulation at first took place among volunteers and enthusiasts from the computer world. Interest grew to the point that bitcoin began to trade in 2010 on a Japanese-based online exchange, Mt. Gox, which had originally been created as a platform for exchanging trading cards from the fantasy game Magic. On the first day of trading on Mt. Gox, 20 bitcoins changed hands at a price of 4.951 cents, for total volume of slightly less than one U.S. dollar.

The first purchase of goods and services using bitcoin is said by Wallace (2011) and other sources to have been two pizzas procured at a cost of 10,000 bitcoins in 2009. The pizza parlor did not accept bitcoins directly, and instead a third-party broker was enlisted who agreed to procure the pizzas using a credit card (based on a real currency) and accept the bitcoins, worth almost $\$ 5$ million at recent prices, as consideration. Much of the commerce involving bitcoins continues to take place using middlemen who facilitate immediate exchanges of bitcoins into conventional currencies.

The Silk Road marketplace, an Internet portal for the sale of illegal narcotics which accepted only bitcoins for payment, was sometimes reported to account for as much as half of the early bitcoin transaction volume although this estimate is subject to considerable dispute. The Silk Road association helped give bitcoins an early reputation for lawlessness, and this outlaw cachet may not have harmed its appeal at all. Bitcoin usage spread into the bricks-andmortar economy, and Silk Road was shuttered by U.S. authorities after they arrested its operator 
in San Francisco in October 2013. Far from hurting bitcoin, this event generated publicity that may have boosted its popularity. Later in the same month, the first bitcoin ATM went into use in a Vancouver coffee shop.

Trading of bitcoins grew rapidly on the Mt. Gox exchange and other platforms. Many online exchanges have now opened to trade bitcoin alongside other virtual currencies that have sprung up as rivals, ${ }^{3}$ though some of these platforms are thinly capitalized and trading can appear episodic at best. As bitcoin's value has increased, exchanges have become targets of hackers; Mt. Gox in April 2013 reported three denial of service attacks that sharply reduced trading volume on various dates, though in each case the exchange appeared to recover in a matter of hours. A number of investment funds have opened to cater to bitcoin speculators, including one that attempted to register with the Securities and Exchange Commission in July 2013 under sponsorship of the Winklevoss twins, who have became famous in the world of digital entrepreneurship due to their legal battles with Mark Zuckerberg over ownership of Facebook.

Bitcoin appeals to two distinct clienteles. One group consists of technology enthusiasts who embrace bitcoin for online commerce. As more and more routine business transactions migrate online, these users believe bitcoin's value should increase due to transaction demand, and they also cite its cost advantages over credit cards and other payment systems for routine bricks and mortar retail shopping. A separate group with pseudo-Libertarian political beliefs finds bitcoin attractive due its lack of connection to any government. Some of these adherents openly distrust the world financial system, and the timing of bitcoin's introduction, coinciding

\footnotetext{
${ }^{3}$ See http://coinmarketcap.com/ for a registry of more than 200 alternative digital currencies, which are being created almost by the day. Currently bitcoin has about $80 \%$ of the world market value of this asset class, according to pricing information on this website.
} 
with the very bottom of the global financial crisis in 2008-09, probably helped swell their ranks.

The technology and libertarian clienteles are united by their enthusiasm for bitcoin but not much else. Discussions about the merits of bitcoin can attract odd mixes of entrepreneurs, academics, and polemicists. An example occurred at a March 2014 panel discussion moderated by a New York Times reporter. In response to a conjecture that up to $10 \%$ of the bitcoin supply has already been pilfered by computer hackers, bitcoin entrepreneur Andreas Antonopoulos called the 10\% theft "a vast improvement over the rest of our economy, where $80 \%$ is in the hands of criminals - and that's the banks." ${ }^{4}$ As the audience applauded in approval, the famous Stanford economics professor Susan Athey looked on from the other side of the stage with a mix of exasperation and bemusement.

The daily transaction flow of bitcoin trades suggests that the large majority of worldwide demand originates in two countries, the U.S. and China. While China has taken various steps to ban the use of bitcoin, U.S. regulators have had a more benign attitude. The relative tolerance of bitcoin by American regulators may stem from their recognition that a universal online audit trail exists for bitcoin transactions. Although services such as "tumblers" exist on the Internet that purport to cloak bitcoin transfers in anonymity, confidence in the security of these protocols appears to be naive. The arrest of Silk Road's operator in October 2013, which took place amid widespread publicity of Internet data monitoring by the U.S. National Security Agency, disabused many about the possibility of keeping any bitcoin-related information anonymous. Tax evasion, money laundering, purchases of contraband, and other illicit activities using online transfers become far riskier when the use of a virtual currency like bitcoin can be reconstructed

\footnotetext{
${ }^{4}$ See http://www.coindesk.com/despite-challenges-bitcoin-technology-stay.
} 
by governments that have sufficient technical skills.

\section{Bitcoin's weaknesses as a currency}

This section presents analyses of ways in which bitcoin fails to conform to the classical properties of a currency. A successful currency typically functions as a medium of exchange, a unit of account, and a store of value. Bitcoin faces challenges in meeting all three of these criteria.

\section{A. Medium of exchange}

Because bitcoin has no intrinsic value, its worth ultimately hinges upon its usefulness as a currency in the consumer economy. Evidence of bitcoin's footprint in daily commerce is mostly anecdotal, consisting of newspaper stories about people living only by spending bitcoin or estimates of large numbers of businesses that are willing to accept bitcoin. To date, only one established business of any size has begun to take bitcoin, the online retailer Overstock.com. ${ }^{5}$ Most of the rankings of the top merchants accepting bitcoins are dominated by computer software and hardware companies selling products narrowly focused on bitcoin applications, and by marketplaces or exchanges providing investor services to bitcoin speculators. ${ }^{6}$

Realistic insight into the adoption of bitcoin can be obtained from data drawn from the universal ledger of bitcoin transactions. According to data available at numerous websites, the

\footnotetext{
5 A recent article estimated Overstock.com's daily bitcoin revenue at about $\$ 30,000$. See http://techcrunch.com/2014/03/12/overstocks-bitcoin-purchases-account-for-less-than-1-of-revenue-but-its-growing/

${ }^{6}$ The most widely cited ranking of the top bitcoin merchants appears to be "The Bitcoin Ladder," https://en.bitcoin.it/wiki/Bitcoin_Ladder, but it is badly out of date, as it ranks the defunct and notorious Mt. Gox and Silk Road as the top two worldwide merchants.
} 
recent bitcoin transaction count has peaked at daily volumes of approximately $70,000{ }^{7}$

However, it is widely understood that most of these transactions involve transfers between speculative investors, and only a minority are used for purchases of goods and services. For instance, Fred Ersham, co-founder of Coinbase, the leading digital wallet service, estimated in a March 2014 interview that $80 \%$ of activity on his site was related to speculation, down from perhaps $95 \%$ a year earlier (Goldman Sachs, 2014). If we take this estimate as correct, then perhaps 15,000 bitcoin transactions per day involve the purchase of a product or service from a merchant. In a world with 7,000,000,000 consumers, most of whom make multiple economic transactions each day, bitcoin appears to have a extraordinarily negligible market presence. Ersham further states in his interview that 24,000 merchants are registered with Coinbase. ${ }^{8}$ If all worldwide bitcoin commerce occurred within this group (almost certainly an exaggeration), these businesses would average well below one transaction per merchant per day. In other words, bitcoin transactions appear to be rarities, even for the small number of merchants that accept them.

One obstacle to bitcoin becoming a widely used medium of exchange arises from the difficulty of procuring new bitcoins. Unless a consumer is successful as a bitcoin miner (an activity now dominated by supercomputers requiring massive capital investments), he or she must source bitcoins from online exchanges or dealers and then find a way to store them securely. These purchases typically cannot be made using a credit card or PayPal, and instead

\footnotetext{
${ }^{7}$ See, for instance, https://blockchain.info/charts/n-transactions.

${ }^{8}$ The co-founder of bitcoin payment processor BitPay estimated the number of worldwide businesses at 26,000 in a separate interview given contemporaneously. See http://money.cnn.com/2014/03/17/smallbusiness/bitcoin-bitpay.
} 
the buyer must make a bank transfer or link an existing bank account to the exchange. ${ }^{9}$ The existing bitcoin exchanges are often have low liquidity, significant bid-ask spreads, and a certain amount of execution and custody risk.

Finally, one cannot bypass the requirement of possessing bitcoins before procuring goods and services from a merchant. Being able to purchase goods without cash in hand occurs routinely in most retail markets, as customers frequently buy with consumer credit financed by the merchant or a third-party credit card vendor. These options are not available for bitcoins, as no bitcoin-denominated credit cards have yet been issued and consumer loans denominated in bitcoin appear to be unheard-of.

\section{B. Unit of account}

For a currency to function as a unit of account, consumers must treat it as a numeraire when comparing the prices of alternative retail goods. For instance, a cup of coffee that costs $\$ 4.00$ in one café is quickly understood to be twice as expensive as a cup of coffee selling for $\$ 2.00$ at another café down the street.

Bitcoin faces a number of obstacles in becoming a useful unit of account. One problem arises from its extreme volatility, an issue discussed in further detail below. Because the value of a bitcoin compared to other currencies changes greatly on a day-to-day basis, retailers that accept the currency have to recalculate prices very frequently, a practice that would be costly to the merchant and confusing to the consumer. In principle this issue would recede in an economy that used bitcoin as its principal currency, but no such place exists in today's world.

\footnotetext{
${ }^{9}$ See http://howtobuybitcoins.info/us.html.
} 
A related problem stems from the diversity of "current market prices" that one can obtain for bitcoin at any given time. For instance, at the moment of writing this paragraph, I consulted a widely used website that posts the prices of bitcoins on markets around the world. The five exchanges with the highest trading volume quoted U.S. dollar prices for one bitcoin of \$454.81, $\$ 453.60, \$ 462.12, \$ 450.84$, and $\$ 480.15$, all for trades having taken place within the several minutes. This disparity of market values, ranging by almost $7 \%$ between the high and low quotes, is a clear violation of the classical law of one price, and it would be unthinkable for these conditions to persist in a developed currency market due to the ease of arbitrage. The uncertain market value of one bitcoin presents a conundrum for any third party vendor or customer seeking to establish a valid reference point for setting consumer prices. As a result, many websites have taken to relying upon unwieldy price aggregations, such as the average bitcoin price over several exchanges over the past 24 hours, but these aggregates do not indicate to merchants and consumers the true cost of procuring or selling a bitcoin at the present time.

Perhaps the most serious obstacle to bitcoin becoming a widely used unit of account and one often overlooked or trivialized by bitcoin enthusiasts - occurs due to the relatively high cost of one bitcoin compared to most ordinary products and services. This requires merchants to quote bitcoin (BTC) prices for most goods in four or more decimal places. Although the mathematics are straightforward, for consumers these decimal points are likely to be disconcerting. For instance, a visit to one online food retailer yields offers of a jar of salsa for 0.01694 BTC, chocolate bars for 0.00529 BTC, and a tea variety pack for 0.05255 BTC. Alternatively, these prices could be expressed in scientific notation as $1.694 \times 10^{-2}$ BTC, $5.29 \mathrm{x}$ $10^{-3} \mathrm{BTC}$, and $5.255 \times 10^{-2} \mathrm{BTC}$, respectively. It is hard to find any other currency in the world 
for which consumer prices are quoted in these units, and indeed, many widely used accounting software products can only accommodate two decimal points in the price of a good.

To the extent that some economies have retail prices that depart from ordinary integers, the pattern usually occurs in the opposite direction as a result of high inflation. Italy prior to the introduction of the euro would be a familiar example for many readers; an ice cream cone from a street vendor in Rome in the 1990s might have cost 5,000 lira, for instance, and consumers generally adapted to these prices by ignoring the three zeroes on the end.

A lengthy literature in marketing research has identified heuristics in consumer pricing that include various integer, left-digit, and right-digit reference points. For example, many goods have prices ending in the digit 9. For a review of this research, see Thomas and Morwitz (2009). One issue in this literature is the difficulty of computations for consumers in comparing the prices of goods. In the example above, the tea is about 10 times as expensive as the chocolate bars, but even consumers with university degrees would often fail to reach this conclusion with ease due to the length of the decimals and the presence of leading zeros.

Bitcoin proponents tend to dismiss the currency's failure to generate prices that line up with ordinary consumer reference points. Sources tend to agree that existing computational data arrays permit division of bitcoins into eight decimal places, providing the potential for a large enough number of units. ${ }^{10}$

\section{C. $\quad$ Store of value}

${ }^{10}$ See, for instance, https://en.bitcoin.it/wiki/Myths, which recommends that "As the value of the unit of 1 BTC grows too large to be useful for day to day transactions, people can start dealing in smaller units, such as milli-bitcoins (mBTC) or micro-bitcoins ( $\mu$ BTC)." 
When currency functions as a store of value, the owner obtains the currency at a certain time and exchanges it for goods and services at some future time of his choice. When the currency is spent, the owner expects to receive the same economic value that the currency was worth when he acquired it.

Throughout much of history, treating currency as a store of value essentially meant protecting it against theft, either by physically hiding it or by putting it into a bank (which then assumed the security problem). Strategies for hiding bitcoins under mattresses or elsewhere cannot work, because the currency has no physical manifestation. Instead, bitcoins must be held in computer accounts known as "digital wallets," and security for these wallets has become a major difficulty for the bitcoin industry. Some digital wallet companies have contracted with third-party insurers to provide a crude form of deposit insurance. While this strategy might work in principle, it forces the customer to bear the cost of evaluating the security (financial and otherwise) of both the wallet company and the insurance company.

If a consumer finds a successful way to hold and secure his bitcoins, he faces the further problem of managing the risk arising from bitcoin's volatility. Figure 2 shows the volatility of the bitcoin-dollar exchange rate, calculated using daily data from 2013. For comparison purposes the graph shows the volatilities of the exchange rates of the Euro, Yen, British Pound, and Swiss Franc as well as the London price of gold, with all volatilities annualized. Bitcoin's exchange rate volatility in 2013 was $142 \%$, an order of magnitude higher than the exchange rate volatilities of the other currencies, which fall between $7 \%$ and $12 \%$. Gold, which is a plausible alternative to these currencies as a store of value, had volatility of $22 \%$ in 2013 based on its dollar-denominated exchange rate. For comparison purposes, most widely traded stocks have 
volatilities in the range of $20 \%$ to $30 \%$, and even very risky stocks rarely exhibit volatility as high as $100 \%$. From Figure 2, one must conclude that holding bitcoins even for a short period is quite risky, which is inconsistent with a currency acting as a store of value and which greatly undermines the ability of a currency to function as a unit of account.

I study the movement of bitcoin compared to the other currencies and to gold in the correlation matrix shown in Table 1. The table is based on the daily changes in the London gold price and each currency's exchange rate against the U.S. dollar, using daily data from July 2010 (the inception of trading on the Mt. Gox exchange) up to the present. For weekend dates when the exchange markets are closed, I use Friday-to-Monday returns for bitcoins and ignore the intervening price changes on Saturdays and Sundays. As shown in the table, the three European currencies tend to exhibit strong positive correlation, with the Euro having 0.61 correlation with the Swiss Franc and 0.64 correlation with the British Pound, and the Pound and Franc having 0.42 correlation. The Yen's exchange rate is also positively correlated with those of the other currencies, albeit at a somewhat reduced level. The same is true of the price of gold. In contrast, the bitcoin-dollar exchange rate exhibits almost zero correlation with the exchange rates of any of the four currencies or with the price of gold.

Bitcoin's complete separation from other prominent international currencies and from gold seems telling. Macroeconomic events that cause similar impacts on the value of various currencies do not seem to affect bitcoin either positively or negatively. The data imply that bitcoin is completely ineffective as a tool of risk management, which is a common use for currencies, and conversely, that it is very difficult to hedge any risks that might affect bitcoin itself. I examined the trading histories of all U.S. stocks in 2011-12 to see whether bitcoin could 
be hedged by selling short individual equities. Using monthly returns from the 2011-12 time period, I found that bitcoin was most closely correlated with Vitamin Shoppe, a retail growth stock with a market capitalization of just under $\$ 2$ billion (Vitamin Shoppe does not appear to accept bitcoin for transactions on its website). Therefore, to best limit exposure to bitcoin, one would have succeeded up to now by selling short the shares Vitamin Shoppe.

\section{Conclusion: Obstacles faced by bitcoin}

For bitcoin to become more than a curiosity and establish itself as a bona fide currency, its daily value will need to become more stable so that it can reliably serve as a store of value and as a unit of account in commercial markets. The excessive volatility shown in Figure 2 is more consistent with the behavior of a speculative investment than a currency. As described above, bitcoin also faces difficulties due to its unorthodox decimal pricing of common household goods, the scarcity of merchants who accept it, and the cumbersome process of procuring bitcoins from a vendor, among other issues. The relatively high level of computer knowledge required for using bitcoins represents a further barrier to bitcoin's wide adoption.

Moreover, bitcoin's legitimacy as a currency should also hinge on its integration into the web of international payments and risk management transactions. Even though it is not issued by a sovereign state, bitcoin imparts risk to any business that accepts it for transactions, just like all other currencies. Major companies that deal in more than one currency, such as multinationals, attempt to hedge themselves against risks related to changes in those currencies' values. Data shown in Table 1 suggest that no effective way exists to hedge bitcoin against the value of other currencies, and the absence of any swap, forward, or other derivative markets for 
bitcoin exacerbates this problem. Bitcoin transactions also are risky due to the absence of basic consumer protection, such as the provision of refunds that result from disputes between merchants and customers. While local laws may provide ground rules for resolving such disputes, because a government has no legal way to foreclose and take possession of bitcoins, it ultimately has little ability to step in and enforce its laws. Similar problems arise in attempting to secure consumer credit denomiated in bitcoin or to pledge bitcoins as collateral for a consumer loan. Again due to its lack of affiliation to any sovereign, bitcoin is ill-suited for use in credit markets because no government can foreclose and seize it in the event of a default.

Bitcoin appears to suffer by being disconnected from the banking and payment systems of the U.S. and other countries. Most currencies are held and transferred through bank accounts, which in turn are protected by layers of regulation, deposit insurance, and international treaties. Without access to this infrastructure, bitcoin has proven vulnerable to fraud, theft, and subversion by skilled computer hackers. However, adherents of bitcoin argue that bitcoin bypasses the well-known flaws in standard financial security systems, which have spawned epidemics of identity theft and related problems for ordinary customers of mainstream businesses.

Finally, bitcoin faces a long-term structural economic problem related to the absolute limit of 21 million units that can ever be issued, with no expansion possible of the bitcoin supply after the year 2140. If bitcoin becomes wildly successful and displaces sovereign fiat currencies, it would exert a deflationary force on the economy since the money supply would not increase in concert with economic growth. This situation would require most workers to accept pay cuts every year, for instance, likely leading to political protests against the currency similar to those 
experienced in the U.S. during the Populist movement at the end of the $19^{\text {th }}$ century. One can imagine a revival of William Jennings Bryan's 1896 "cross of gold" speech in the next century, updated with futuristic rhetoric about the economic tyrrany of an uber-currency with an inflexible supply. 


\title{
References
}

Goldman Sachs Investment Research, 2014, "Interview with Fred Ersham,” Top of Mind 21, 8, March 11.

Nakamoto, Satoshi, 2008, "Bitcoin: A Peer-to-Peer Electronic Cash System," unpublished manuscript, retrieved at http://pdos.csail.mit.edu/6.824/papers/bitcoin.pdf.

Thomas, Manoj and Vicki Morwitz, 2009, "Heuristics in Numerical Cognition: Implications for Pricing" in Vithala R. Rao ed., Handbook of Pricing Research in Marketing (Northampton, MA: Edward Elgar Publishing).

Wallace, Benjamin, 2011, "The Rise and Fall of Bitcoin," Wired, November 23, retrieved at http://www.wired.com/magazine/2011/11/mf_bitcoin.

\author{
Data sources \\ http://bitcoincharts.com/charts/mtgoxUSD (Bitcoin). \\ http://oanda.com/currency/historical-rates/ (currencires) \\ https://research.stlouisfed.org/fred2/ (gold)
}




\section{Figure 1}

Market value of one bitcoin in U.S. dollars

The figure shows the value on a logarithmic scale of the bitcoin-dollar exchange rate, recorded daily at midnight on the Mt. Gox Exchange in Japan from July 17, 2010, up to February 6, 2014, and thereafter until March 21, 2014 on the Bitstamp Exchange.

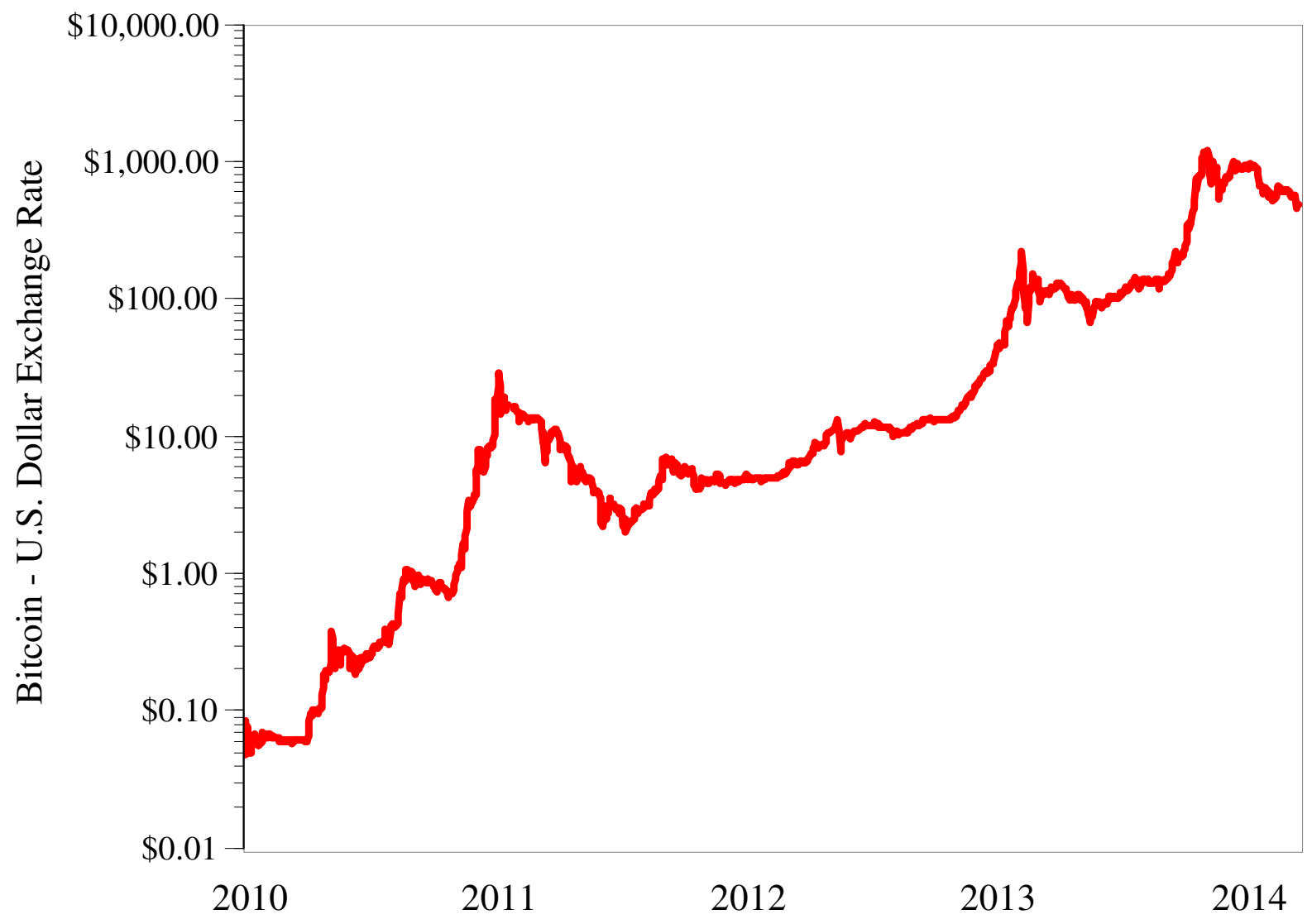




\section{Figure 2}

Volatility of bitcoin compared to major currencies and gold

The figure shows the volatility during the year 2013 of the percentage change in daily exchange rates for four major currencies, gold, and bitcoin, all measured against the U.S. dollar.

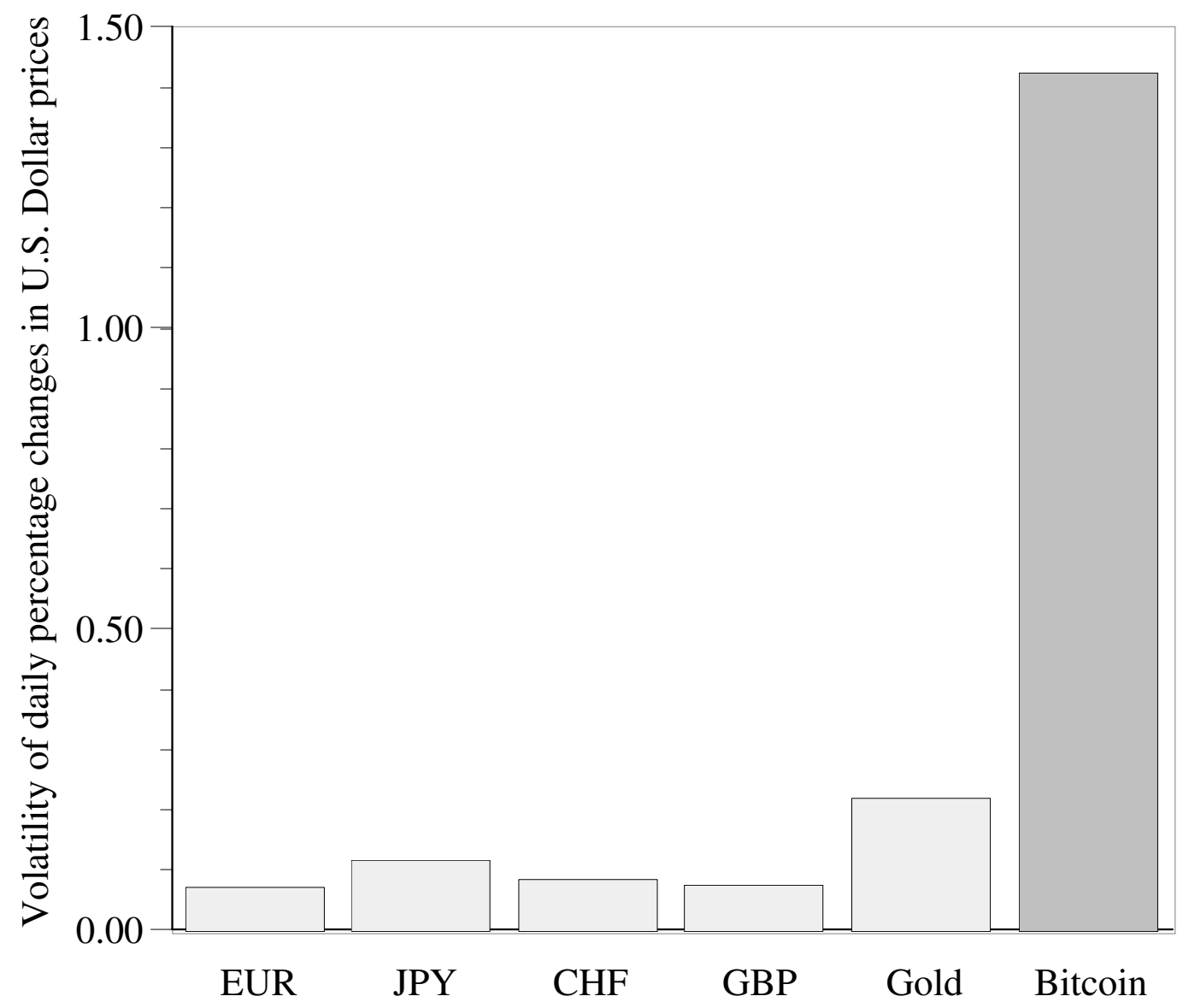




\section{Table 1}

Correlation matrix of daily changes in exchange rates, bitcoin, and gold

The table shows simple correlations of the percentage changes in daily exchange rates for pairs of currencies, with all exchange rates measured against the U.S. dollar. In addition, the table shows correlations between each currency and the percentage change in the daily London gold price as measured in U.S. dollars at 3 p.m. Correlations are calculated for the period from July 19, 2010 up to March 21, 2014.

$\begin{array}{lrrrrrr} & \text { EUR } & \text { JPY } & \text { CHF } & \text { GBP } & \text { Gold } & \text { Bitcoin } \\ \text { EUR } & 1.00 & 0.18 & 0.61 & 0.64 & 0.20 & -0.05 \\ \text { JPY } & & 1.00 & 0.33 & 0.20 & 0.07 & 0.01 \\ \text { CHF } & & & 1.00 & 0.42 & 0.19 & -0.04 \\ \text { GBP } & & & & 1.00 & 0.21 & -0.02 \\ \text { Gold } & & & & & 1.00 & -0.06 \\ \text { Bitcoin } & & & & & & 1.00\end{array}$

